\title{
Does Coronary Endarterectomy Increase Early Mortality and Morbidity Compared with Coronary Artery Bypass Surgery Alone-Single Centre Experience
}

\author{
Mohammed Alreshidan, Mohammed Albabtain, Hamoud Obied, Mohamed Alassal, \\ Abdulaziz Albaradai, Fahad Alghofaili \\ Cardiac Surgery Department, Prince Salman Heart Centre (PSHC), King Fahad Medical City (KFMC), Riyadh, KSA \\ Email: $\underline{\text { msr555@hotmail.com }}$
}

Received 22 December 2013; revised 21 January 2014; accepted 20 February 2014

Copyright (C) 2014 by authors and Scientific Research Publishing Inc.

This work is licensed under the Creative Commons Attribution International License (CC BY). http://creativecommons.org/licenses/by/4.0/

(c) (i) Open Access

\begin{abstract}
Background: With increasing incidence of coronary artery disease (CAD) in Saudi Arabia and its fatal co-morbidity, this has resulted in a more complex pool of cases referred to cardiac surgeons. In selected cases, achieving optimal revascularization of diffuse CAD necessitates adjunctive techniques such as coronary endarterectomy (CE). The aim of our study is to evaluate the early mortality and post-operative morbidity related to CE. Method: Retrospective study from Jan 2009 to Dec 2010. Inclusion of 186 patients: 38 patients had CABG with CE and 148 patients had CABG alone. Results: Pre-operative percutaneous coronary intervention (PCI) was higher in the CE with CABG group (25.8\%) compared with CABG alone $(8.9 \%)(p=0.009)$. Intra-operative data showed a higher mean cardiopulmonary bypass time (CBT) of 160 minutes and a mean cross-clamp time (XCT) of 109 minutes in the CE with CABG, compared to a mean CBT of 129 minutes and a mean XCT of 87 minutes in the CABG alone group $(p=0.001)$. The most common vessel endarterectomized was left anterior descending artery (LAD) (47\%) followed by right coronary artery (RCA) (22\%). Post-operatively, the mortality amongst the CE with CABG group (7.9\%) was higher than CABG only group $(1.4 \%)$, however it wasn't statistically significant $(p=0.06)$. With regards to morbidity, hemodynamic instability requiring intra aortic balloon pump (HIR-IABP) was higher in the CE with CABG group $(10.5 \%)$ compared to the CABG only group $(1.4 \%)(p=0.018)$. Conclusion: Our study showed that $C E$ when combined with CABG wasn't associated with a higher mortality rate when compared with CABG alone.
\end{abstract}

\section{Keywords}

CABG; Coronary Endarterectomy; Mortality; Morbidity

How to cite this paper: Alreshidan, M., Albabtain, M., Obied, H., Alassal, M., Albaradai, A. and Alghofaili, F. (2014) Does Coronary Endarterectomy Increase Early Mortality and Morbidity Compared with Coronary Artery Bypass Surgery AloneSingle Centre Experience. International Journal of Clinical Medicine, 5, 197-205. http://dx.doi.org/10.4236/ijcm.2014.55033 


\section{Introduction}

Coronary artery disease (CAD) is one of the world's leading causes of death [1]. The prevalence of CAD in Saudi Arabia (SA) is 5.5\% as reported in a survey done in 2004 [2]. With the novel advent of percutaneous coronary intervention (PCI), coronary artery bypass surgery (CABG) cases have become more challenging, with complex anatomical considerations [1] [3] [4]. Furthermore, the increasing incidence of diabetes mellitus (DM) amongst the Saudi population [5], along with other components of metabolic syndrome like obesity and dyslipidemia (DLP), the anatomy of CAD and its surgical approach has become more cumbersome [6]. Treating complex CAD with conventional coronary artery bypass surgery (CABG) yields unsatisfactory results [7]. To achieve complete revascularization in complex CAD, adjunctive surgical techniques like coronary endarterectomy (CE) are necessary [8]. CE was first introduced in 1957 by Bailey et al., and was further corroborated by multiple reports [9]. Early series of CE in the 1960's and 1970's showed a high risk of post operative mortality and morbidity [10] [11], however, with further refinement in the technique, the last three decades have witnessed more favorable outcomes following CE with rates of morbidity and mortality in patients treated with CABG combined with CE approaching those treated with CABG alone [12]-[15]. Moreover, CE has shown to be a valuable adjunct in off pump CABG when treating patients with diffuse CAD [16] [17]. The aim of this study was to determine if CE combined with CABG increase the early mortality and morbidity compared to CABG alone.

\section{Patients and Methods}

The study was a retrospective chart review of patients treated at single Centre (Prince Salman Heart Centre, King Fahad Medical City, Riyadh, Saudi Arabia). Cases were performed between January 2009 to December 2010 were included. Out of a cohort of 324 patients whom underwent cardiac surgery, 186 patients had undergone CABG and CABG with CE. Out of these 186 patients, 38 patients underwent CABG with CE, and 148 patients underwent $\mathrm{CABG}$ alone.

Indications of $\mathbf{C E}$ were:

1) Total occluded vessel that supplies viable myocardium.

2) Significant disease (>75\% narrowing), with multi-segmental narrowing.

3) Severely calcified vessel that makes the anastomosis difficult.

4) Incomplete revascularization with conventional CABG.

Surgical techniques: all CABG operations were performed using a cardiopulmonary bypass machine (CPB) combined with mild hypothermia $32^{\circ} \mathrm{C}$ to $34^{\circ} \mathrm{C}$. CPB was established via the standard fashion of ascending aorta and right atrial cannulation. Cardiac arrest and myocardial protection were achieved by using intermittent cold blood cardioplegic solution delivered at 4:1 ratio, and given in an antegrade manner through aortic root. In certain cases, the myocardial protection was supplemented with retrograde cardioplegia through the coronary sinus to achieve better protection. The distal and proximal anastomoses were done during the cardiac arrest. With regards to CE, it was done by using two techniques, open (direct-vision) or closed (pulled-out) technique, depending on the vessels and the lesion that has been endartectomized. To complete the procedure, adequate homeostasis was achieved, chest tubes were inserted, and the chest was closed in the usual manner.

Open or direct-vision technique: this was done mainly to endartectomize the left anterior descending artery (LAD). After doing the longitudinal arterotomy with the micro-blade, if the vessel was severely diseased and the previously mentioned indications were present, the decision to do endarterectomy was undertaken. The longitudinal arterotomy was extended as needed to make sure all diseased segments were removed. Then with careful manipulation, the intimae and media of the vessel, including the main core of lesion along with its branches, were removed by using a special spatula. Lastly, the vessel was reconstructed by using long saphenous vein patch angioplasty with 7-0 polypropylene, and then attaching the left internal mammary artery (LIMA) or saphenous vein graft to the patch by using 7-0 polypropylene (Figure 1). Alternatively, we reconstructed the LAD using the LIMA on-lay patch angioplasty with 7-0 polypropylene (Figure 2).

Closed or pulled-out technique: was done mainly to endartectomize the right coronary artery (RCA) and the remaining coronary arteries. After doing a longitudinal arterotomy with the micro-blade, if the vessel was severely diseased and the criteria of endarterectomy were matched, the lesion was carefully dissected and separated from the vessel wall by using the spatula. Then gentle traction was applied to the plaque in either direction, proximally and distally. At the same time, counter-traction was applied with forceps to the adventitia of the ves- 

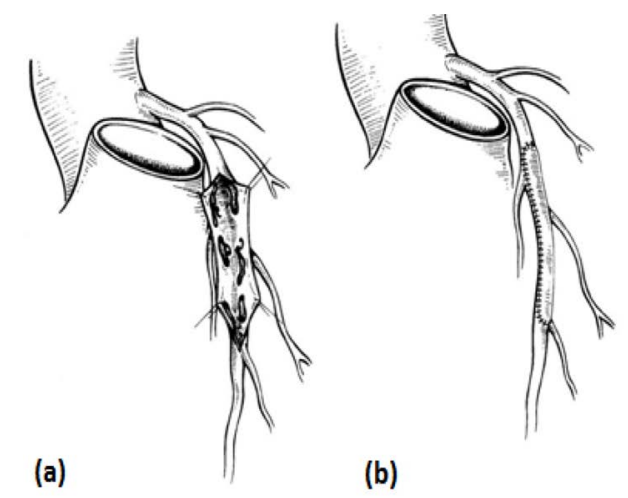

(b)

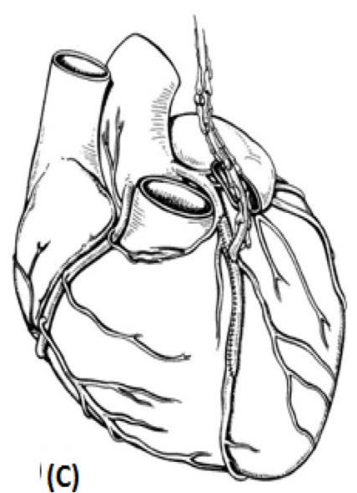

Figure 1. Endarterectomy to left anterior descending artery (LAD). (a) Extended of arterotomy and removal of intimae and media in the diseased segment (plaque); (b) Reconstruction of LAD with saphenous vein patch; (c) Anastomosis the left mammary artery (LIMA) to the vein patch in LAD. Copyright to Oxford University Press 2002.
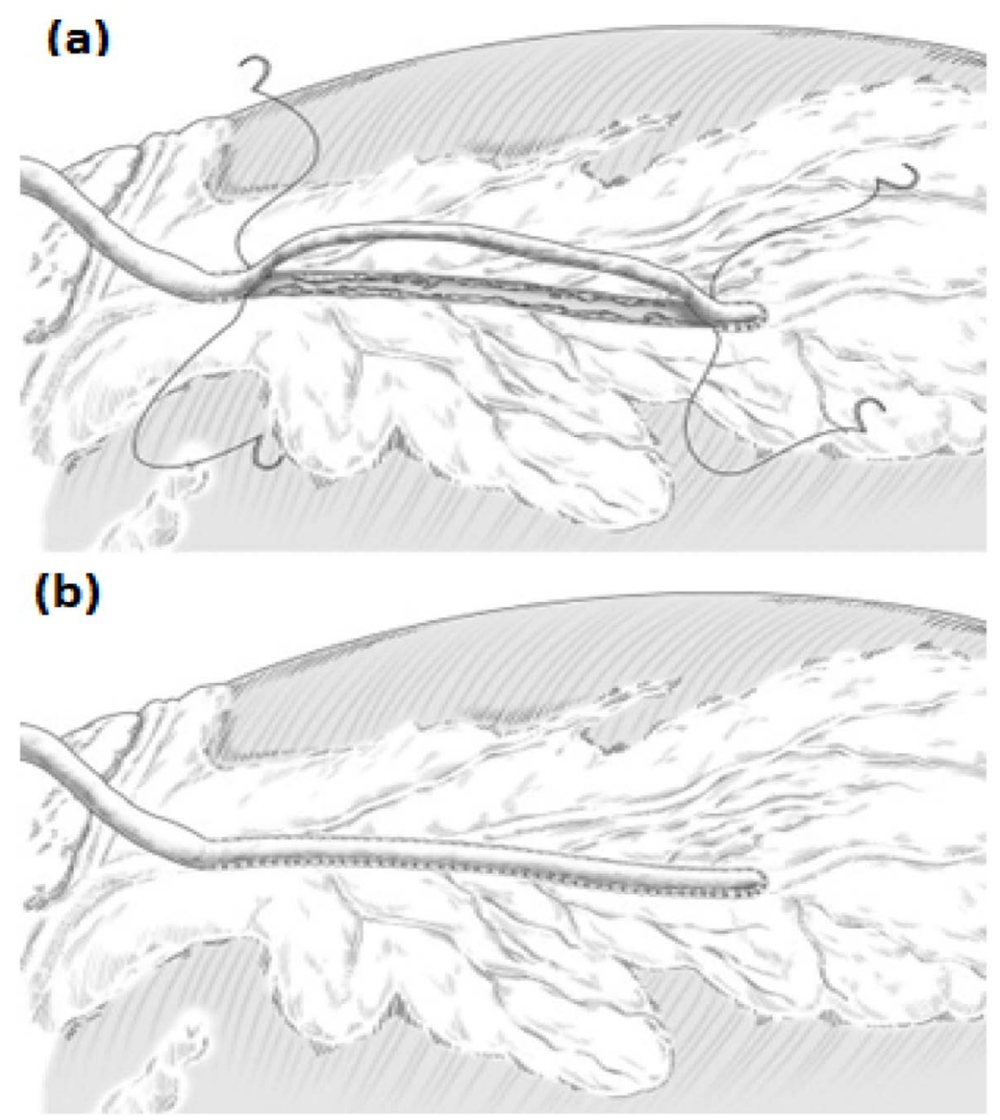

Figure 2. Reconstruction of left anterior descending artery (LAD) with left internal mammary artery (LIMA) on-lay patch. Copyrights to Elsevier Inc. 2011.

sel wall, while pulling and peeling the plaque from the vessel wall. A standard distal anastomosis was then done using saphenous vein graft with 7-0 polypropylene.

Definitions and post-operative care: mortality was defined as death occurring within a period of 30 days postoperatively. Significant bleeding was defined as postoperative bleeding requiring 4 or more units of packed red blood cells (PRBCs), or the need for re-exploration postoperatively. Hemodynamic instability (HI) requiring 
intra aortic balloon pump (IABP) was defined as worsening of hemodynamic parameters postoperatively not responding to intravenous (IV) pressors. Post operative myocardial infarction (MI) referred to MI that was detected by electrocardiogram (ECG) and evident by a significant increase in Troponin I. Sepsis was defined as systematic infection that was detected by blood cultures, requiring IV antibiotic and pressors. Atrial fibrillation referred to irregular rhythm that was detected on ECG, which required treatment. Pneumonia referred to lung infection that was detected by chest x-ray (CXR), and required IV antibiotic. Plural effusion was defined as an accumulation of fluid in the plural space on the CXR, which caused respiratory distress which required insertion of a chest tube. Lastly, sternal wound infection was defined as infection in the wound that required debridement and IV antibiotic. Post-operative care was standardized for all patients which included, Aspirin (80 mg), beta blocker (mainly Metoprolol), and statin (mainly Lipitor) which were all started on day one postoperatively. Patients with CE were treated with Plavix (75 mg) or Warfrain, day one postoperatively, depending mainly on surgeon preference, and the rational of adding a second agent together with the Aspirin was to prevent early activation of the coagulation cascade that has been previously reported post CE [11] [18].

Statistical analysis, data were collected in retrospective manner. Statistical analysis was performed using SPSS version 14 software (SPSS Inc., IBM, Chicago, IL, USA). Chi-square test was used to analyze the categorical data, and continuous numerical data were analyzed by Mann-Whitney tests. The $p$-value of less than 0.05 was considered significant.

\section{The Results}

The results are presented stratified into three main categories: preoperative, intaroperative, and postoperative results.

Pre-operative results: Table 1 shows the preoperative data for the both groups. Both groups were similar in terms of age, gender, and preoperative co-morbidities like DM, hypertension (HTN), dyslipidemia (DLP), and smoking, but previous PCI was higher in the CE group (25\%) compared with CABG only group (8.9\%) with a significant $p$ value of 0.009 . The mean age of the CE with CABG and the CAGB alone group was 61.4 and 60.1 years, respectively, and more than two thirds of the patients were males. More than $75 \%$ of patients in both group had DM, HTN, and DLP. Looking at smoking rate, around half of the patients were smokers in both groups. Preoperative euroscore (ES) and ejection fraction didn't show any difference between the two groups.

Intra-operative results: the cardiopulmonary bypass time (CBT) and cross clamp time (XCT) of both groups are shown in Figure 3. The CBT was 160 minutes in CE with CABG group and 109 minutes in CABG only group, with a significant $p$ value of 0.001 . The XCT time was 129 minutes and 87 minutes in CE with CABG group and CABG only group respectively, and also had a significant $p$ value of 0.001 . Furthermore, the site and number of endartectomized vessels are shown in Figure 4. In our series, the LAD was the most common artery that was endarterectomized (47\%), followed by the RCA (22\%). 36 patients had single vessel endarterectomy. However, 2 patients had double vessel endarterectomy, LAD and obtuse marginal (OM) in one, and LAD and RCA in the other one.

Post-operative results: Table 2 illustrates the 30-day mortality and postoperative morbidity. The mortality amongst the CE with CABG group (7.9\%) was higher than CABG only group (1.4\%), however it wasn't statistically significant $(p=0.06)$. The incidence of myocardial infarction (MI) was $2.6 \%$ in CE with CABG group,

Table 1. Pre-operative characteristics of the patients.

\begin{tabular}{cccc}
\hline Variables & CE + CABG (n = 38) & CABG (control, $\mathbf{n}=\mathbf{1 4 8})$ & $\boldsymbol{p}$-values \\
\hline Age (years) & 61.4 & 60.1 & 0.487 \\
Sex (male) & $32(80.0 \%)$ & $106(72.6 \%)$ & 0.344 \\
Diabetes mellitus (DM) & $27(75.0 \%)$ & $112(81.2 \%)$ & 0.412 \\
Hypertension (HTN) & $28(77.8 \%)$ & $114(82.6 \%)$ & 0.505 \\
Dyslipidemia (DLP) & $26(76.5 \%)$ & $102(74.4 \%)$ & 0.808 \\
Family history of CAD & $9 / 34(26.5 \%)$ & $33 / 136(24.3 \%)$ & 0.79 \\
Smoking & $15 / 34(44.1 \%)$ & $72 / 137(52.6 \%)$ & 0.378 \\
Ejection fraction (EF) & $44.2( \pm 10.2)$ & $45.1( \pm 12.6)$ & 0.694 \\
Previous PCI & $8(25.8 \%)$ & $12(8.9 \%)$ & $\mathbf{0 . 0 0 9}$ \\
Euro score & $7.4( \pm 6.2)$ & $6.7( \pm 6.2)$ & 0.601 \\
\hline
\end{tabular}




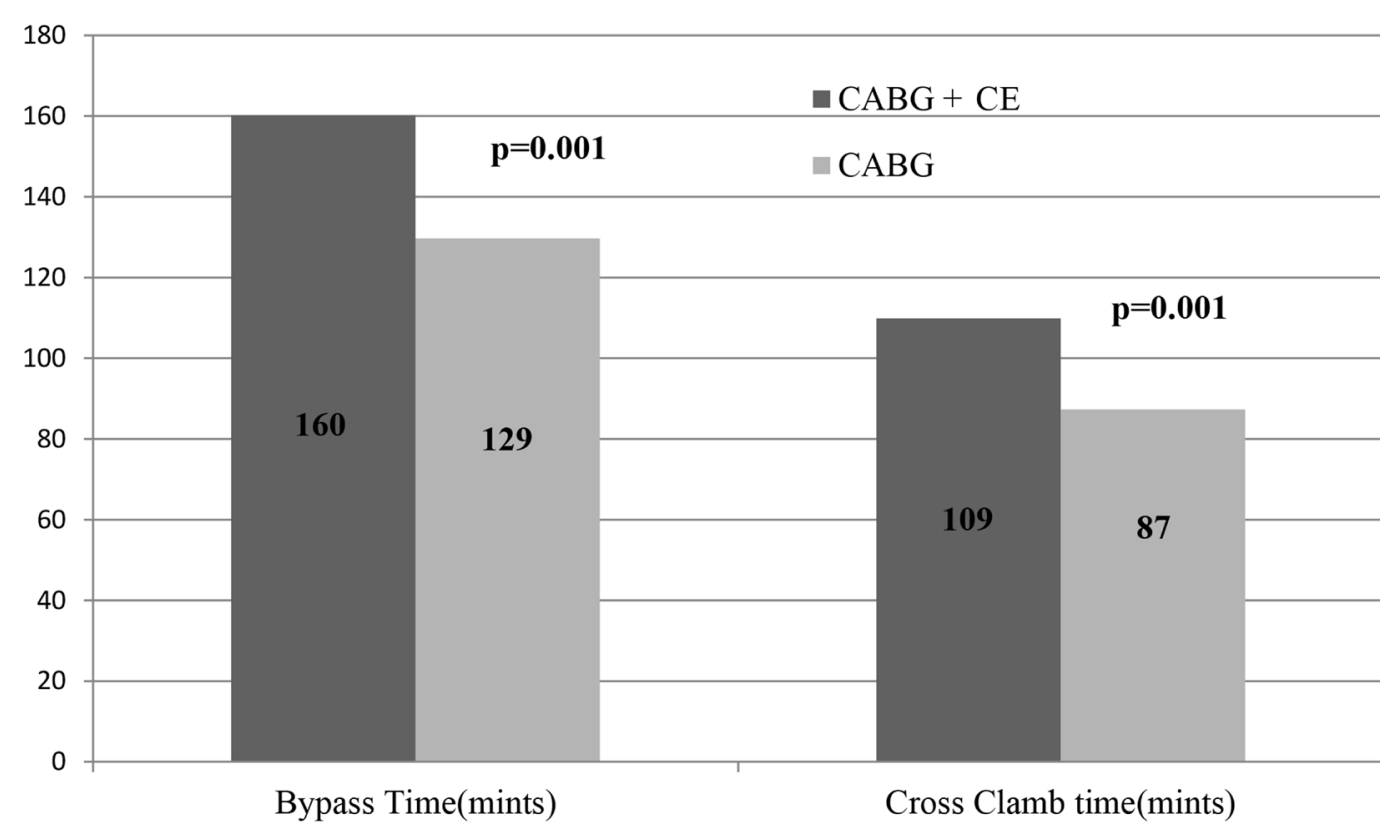

Figure 3. The cardiopulmonary bypass time (CBT) and cross clamp time (XCT), in CE and CABG Group the CBT and XCT were longer than CABG alone, with significant $p$ value respectively $(p=0.001)$.

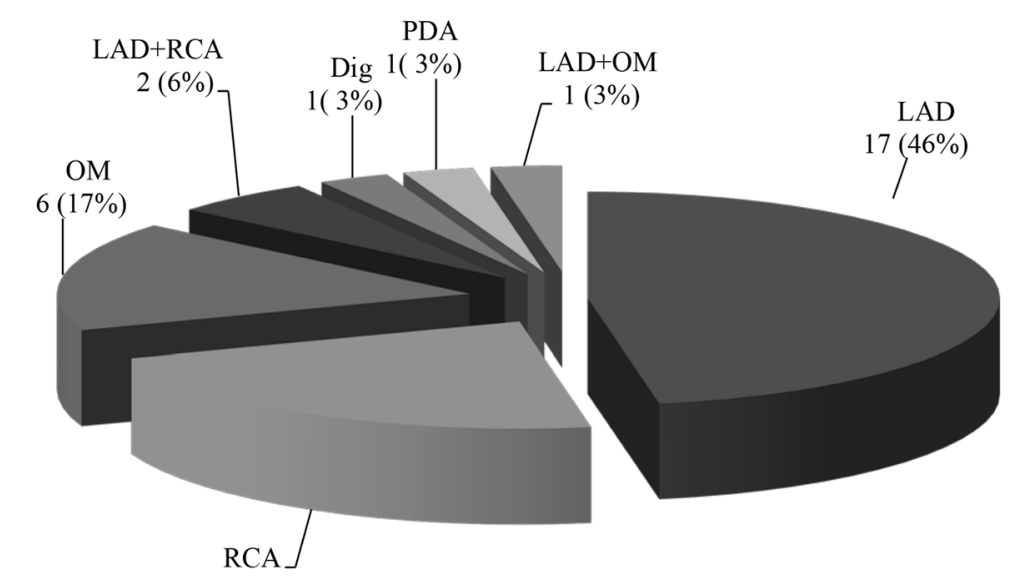

Figure 4. The site and number of endarterctmies vessels. LAD: left anterior descending artery, PDA: posterior descending artery, OM: obtuse marginal artery, Dig: diagonal artery, RCA: right coronary artery.

Table 2. Post-operative 30-day mortality and co-morbidity.

\begin{tabular}{cccc}
\hline Variables & CE + CABG (n = 38) & CABG (control, $\mathbf{n}=\mathbf{1 4 8})$ & $\boldsymbol{p}$-values \\
\hline Mortality & $3(7.9 \%)$ & $2(1.4 \%)$ & 0.06 \\
Myocardial infarction (MI) & $1(2.6 \%)$ & $2(1.3 \%)$ & 0.421 \\
Bleeding & $4(10.5 \%)$ & $7(4.9 \%)$ & 0.246 \\
Hemodynamic instability (required IABP) & $4(10.5 \%)$ & $2(1.4 \%)$ & $\mathbf{0 . 0 1 8}$ \\
Atrial fibrillation & $8(21.1 \%)$ & $15(10.4 \%)$ & 0.082 \\
Pleural effusion & $3(7.8 \%)$ & $6(4.2 \%)$ & 0.351 \\
Pneumonia & $1(2.6 \%)$ & $3(2.1 \%)$ & 0.368 \\
Sepsis & $3(7.8 \%)$ & $7(4.9 \%)$ & 0.542 \\
Sternal wound infection & $1(2.6 \%)$ & $11(7.6 \%)$ & 0.265 \\
\hline
\end{tabular}

IABP: intra aortic balloon pump. 
and $1.3 \%$ in CABG only group, with no statistical significance observed $(p=0.421)$. The CE with CABG group had a higher rate of bleeding, atrial fibrillation (AF), and pleural effusion; however these findings were not statistically significant. Hemodynamic instability that required intra aortic balloon pump (HIR-IABP) was higher in CE with CABG group (10.5\%) compared with CABG only group (1.4\%), with a significant $p$ value ( $p=0.018$ ).

For further assessment of the confounders in the CE with CABG group, we performed subgroup analysis between the mortality cases and survival cases in CE with CABG group (Table 3). The mortality cases were all male and had underlying DM, HTN, DLP, previous PCI, hemodynamic instability that required-IABP (HIR-IABP), and sepsis. With regards to MI, one case in mortality group developed MI compared with survival group, and this showed no statistical significance $(p=0.12)$. The only confounders that were found to be statistically significant were HIR-IABP $(p=0.001)$ and sepsis $(p=0.007)$.

\section{Discussion}

Coronary artery disease (CAD) is a major cause of morbidity and mortality worldwide [1]. In 2004, the prevalence of CAD was 5.5\% amongst our population, with a rapidly increasing incidence [2]. As the life expectancy has risen due to the development in medicine, we witness a congruent increase in the elderly population and their associated co-morbidities [5], which will translate into a higher incidence of complex CAD. Furthermore, with the advent in technology and minimally invasive approaches, diagnostic and interventional modalities like PCI have served the population with simple CAD, leaving the complex cases behind to the cardiac surgeon to reconstruct [1] [3] [4]. Looking at our cohort of patients referred for CABG, more than $75 \%$ of patients had DM, hypertension and dyslipidemia, and even more, $10 \%$ of them had previous PCI. With more diffuse CAD, complete revascularization becomes an issue with conventional CABG, hence the need to adopt alternative surgical techniques such as coronary endareterctomy (CE) [8].

CE was first performed in 1957, with early reports demonstrating discouraging outcomes [9]-[11]. However, the growth of evidence on safety and efficacy of CE combined with CABG made it more acceptable approach to tackle diffuse CAD [12]-[15]. A careful selective approach should be adopted to guarantee the best results. Tiruvoipati et al. have reported the most important factors in determining the ideal patient for endarterectomy which include: 1- diffuse CAD, 2- diseased artery with viable myocardium, and 3- inability to perform distal vessel anastomoses [19]. In our study we adopted strict criteria in identifying patient eligibility for CE which are: total occluded vessel that supplies viable myocardium, significant disease ( $>75 \%$ narrowing) with multi-segmental narrowing, severely calcified vessel that makes the anastomosis difficult or incomplete revascularization with conventional CABG. CE is technically challenging, and has shown to significantly increase cardiopulmonary bypass time (CBT) and cross clamp time (XCT) [15] [20]. Christakis et al. reported a mean CBT of $107 \mathrm{~min}$ for CE with CABG, and $99 \mathrm{~min}$ for CABG alone group $(p=0.001)$, and a mean XCT of $60 \mathrm{~min}$ in CABG with CE and 48 min with CABG alone $(p=0.001)$ [7]. Another study done in Japan by Kato et al. demonstrated that CE with CABG had a mean CBT time of $198 \mathrm{~min}$ and a mean XCT of 143 min, compared with CBT of 161 min and XCT of 113 min in CABG alone $(p=0.001)$ [12]. In our cohort, CE with CABG had a mean CBT of 160 min and a mean XCT of 109 min compared with CABG alone that had CBT of 129 and XCT of $87(p=0.001)$, further corroborating the data reported in the literature.

Table 3. Comparison between mortality cases and survival cases in CE and CABG group.

\begin{tabular}{cccc}
\hline Variable & Mortality CE + CABG (3 patients) & Survival CE + CABG (35 patients) & $p$ Value \\
\hline Age & $67( \pm 3.4)$ & $61( \pm 13.1)$ & 0.212 \\
Sex (Male) & $3(100 \%)$ & $29(78.3 \%)$ & 0.502 \\
MI & $1(33.3 \%)$ & 0 & 0.120 \\
DM & $3(100 \%)$ & $28(75.6 \%)$ & 0.455 \\
HTN & $3(100 \%)$ & $26(4.2 \%)$ & 0.433 \\
Family History of CAD & $3(100 \%)$ & $8(24.2 \%)$ & 0.265 \\
Previous PCI & $3(100 \%)$ & $7(20 \%)$ & 0.258 \\
HIR-IABP & $3(100 \%)$ & $1(2.9 \%)$ & $\mathbf{0 . 0 0 1}$ \\
AF & $1(33.3 \%)$ & $7(20 \%)$ & 0.519 \\
Sepsis & $3(100 \%)$ & 0 & $\mathbf{0 . 0 0 7}$ \\
\hline
\end{tabular}

MI: myocardial infarction, DM: diabetes mellitus, HTN: hypertension, PCI: percutaneous coronary intervention, HIR-IABP: hemodynamic instability required-intra aortic balloon pump, AF: atrial fibrillation. HIR-IABP and sepsis were statistically significant with $p=0.001$ and $p=0.007$, respectively. 
Two CE techniques have been described, open (direct-vision) and closed (pulled-out) [21]. In our centre, the open technique was done mainly for LAD endarterectomy with either vein patch angioplasty or on-lay LIMA angioplasty, to ensure that the distal run off is as adequate as possible. The closed technique was done for RCA and other coronary vessels, reflecting the common practice that has been described in the literature [21]. Shapira et al., at Boston University School of Medicine demonstrated that the closed technique was used for both RCA and LAD, but if a LAD lesion was very irregular, and the distal run off was not satisfactory, the arteriotomy was extended and the open technique was carried out [6]. In a study at Harvard Medical school, Schimitto et al. reported that the open technique was used in $79 \%$ of total endarterectomy cases [22]. In early series, CE was done mainly for RCA lesions because of its large caliber making the procedure technically easier, with minimal complications [21]. Although, LAD endarterectomy carried a higher risk of mortality in early studies [23], recent studies have reported similar risks in LAD endarterectomy compared with other vessels endarterectomy [21]. Schimitto et al., reported an early mortality rate of 5\% in 104 patients, with most common vessel being endactectomized was the RCA (55\%) followed by LAD (35\%) [22]. However, Abid et al., demonstrated an early mortality rate of $2 \%$ (cases: RCA endarterectomy $44 \%$, and LAD endarterectomy $42 \%$ ), and there was no statistical difference in mortality when compared with CABG alone [8]. Moreover, Silberman et al., showed a mortality rate with $\mathrm{LAD}$ endarterectomy reaching $10 \%$, compared with non-LAD endarterectomy (4\%) and CABG alone (3\%) with significant $p$ value of 0.001 [20]. In our study, LAD was the most common endarterectomized vessel (47\%), followed by RCA (22\%), overall mortality was higher in the CE with CABG group (7.9\%) compared with CABG alone (1.4\%); however this was not statistically significant.

Conflicting evidence holds regarding post-operative complications of CE and CABG compared to CABG alone [11] [20] [24]. In our cohort, half of the patients had LAD endarterectomy, and this did not translate into a higher post-operative MI nor early mortality when compared to other vessel endarterectomy. In our study, CE combined with CABG showed a non significant higher incidence of postoperative bleeding (10.5\%) compared with CABG alone (4.9\%). Christakis et al. demonstrated a rate of bleeding in CE with CABG of 2.2\% ( $\mathrm{n}=317)$, compared with $1.6 \%$ in CABG alone $(n=911)$ without statistical significance $(p=0.58)$ [7]. In our cohort, the higher bleeding rate with CABG and CE could be explained by many reasons: platelets dysfunction related to longer CBT [25], longer arterotomy for LAD reconstruction, and the use of dual antiplatelet therapy such as Aspirin and Plavix could also be related. Moreover, the CE and CABG group had more intra aortic balloon pump (IABP) application compared to the CABG alone group $(p=0.018)$, however, in the literature the CE combined with CABG wasn't associated with higher incidence of IABP [6] [7] [26]. Furthermore, after doing subgroup analysis, we found again significant difference between mortality cases of CE with CABG (100\%), compared with survival cases of CE with CABG $(2.9 \%)$ with $(p=0.001)$, this finding again could support what has been shown in multivariate analysis [20]. In our subgroup analysis, we found significant differences in sepsis rates and the need for IABP when comparing patients who passed away vs. patients who survived (Table 3). Higher sepsis rates with CE with CABG cases that passed away can be explained by long ICU stay and aggressive measures.

Limitations of our study include the retrospective design and its inherent flaws in complete data acquisition, a small sample size, and the definitions of overall outcomes being limited to hospital outcome and early 30-day mortality. To the best of our knowledge, this is the first report on CE outcomes in Saudi Arabia, however a longer follow up is required to ascertain the long-term patency of these techniques.

\section{Conclusion}

We conclude that in selected patients, CE combined with CABG provides promising outcomes when compared with CABG alone. Patients who undergo CE carry a higher risk profile, and are relatively more prone to a complicated post-operative course compared to those patients who are eligible for CABG only. In CE patients, the post-operative events and mortality can be attributable to higher rates of IABP and sepsis. The conflicting evidence on the results of CE in conjunction with CABG urges for a standardized approach in order to stratify patients to the optimal myocardial revascularization procedure.

\section{References}

[1] Ariyaratnam, P., et al. (2012) Long-Term Survival from 801 Adjunctive Coronary Endarterectomies in Diffuse Coronary Artery Disease. European Journal of Cardio-Thoracic Surgery, 42, e140-e145. 
http://dx.doi.org/10.1093/ejcts/ezs510

[2] Al-Nozha, M.M., et al. (2004) Coronary Artery Disease in Saudi Arabia. Saudi Medical Journal, 25, 1165-1171.

[3] Fukui, T., et al. (2011) Extensive Reconstruction of the Left Anterior Descending Coronary Artery with an Internal Thoracic Artery Graft. Annals of Thoracic Surgery, 91, 445-451. http://dx.doi.org/10.1016/j.athoracsur.2010.10.002

[4] Santini, F., et al. (2002) Mid-Term Results after Extensive Vein Patch Reconstruction and Internal Mammary Grafting of the Diffusely Diseased Left Anterior Descending Coronary Artery. European Journal of Cardio-Thoracic Surgery, 21, 1020-1025. http://dx.doi.org/10.1016/S1010-7940(02)00074-X

[5] Al-Nozha, M.M., et al. (2004) Diabetes Mellitus in Saudi Arabia. Saudi Medical Journal, 25, 1603-1610.

[6] Shapira, O.M., et al. (1999) Improved Clinical Outcomes in Patients Undergoing Coronary Artery Bypass Grafting with Coronary Endarterectomy. Annals of Thoracic Surgery, 68, 2273-2278. http://dx.doi.org/10.1016/S0003-4975(99)01050-4

[7] Christakis, G.T., et al. (1993) Does Coronary Endarterectomy Adversely Affect the Results of Bypass Surgery? Journal of Cardiac Surgery, 8, 72-78. http://dx.doi.org/10.1111/j.1540-8191.1993.tb00577.x

[8] Abid, A.R., et al. (2009) Hospital Outcome of Coronary Artery Bypass Grafting and Coronary Endarterectomy. Asian Cardiovascular and Thoracic Annals, 17, 59-63. http://dx.doi.org/10.1177/0218492309102609

[9] Bailey, C.P., May, A. and Lemmon, W.M. (1957) Survival after Coronary Endarterectomy in Man. Journal of the American Medical Association, 164, 641-646. http://dx.doi.org/10.1001/jama.1957.02980060017005

[10] Asimakopoulos, G., Taylor, K.M. and Ratnatunga, C.P. (1999) Outcome of Coronary Endarterectomy: A Case-Control Study. Annals of Thoracic Surgery, 67, 989-993. http://dx.doi.org/10.1016/S0003-4975(99)00094-6

[11] Binsalamah, Z.M., et al. (2013) Mid-Term Outcome and Angiographic Follow-Up of Endarterectomy of the Left Anterior Descending Artery in Patients Undergoing Coronary Artery Bypass Surgery. Journal of Cardiac Surgery, 29, 1-7. http://dx.doi.org/10.1111/jocs.12230

[12] Kato, Y., et al. (2012) Results of Long Segmental Reconstruction of Left Anterior Descending Artery Using Left Internal Thoracic Artery. Annals of Thoracic Surgery, 93, 1195-1200. http://dx.doi.org/10.1111/jocs.12230

[13] Khalifa, A.A., et al. (2011) Medium-Term Survival of Diffuse Coronary Artery Disease Patients Following Coronary Artery Reconstruction with the Internal Thoracic Artery. Cardiology, 120, 192-199. http://dx.doi.org/10.1159/000335068

[14] Marinelli, G., et al. (2002) Bypass Grafting with Coronary Endarterectomy: Immediate and Long-Term Results. Journal of Thoracic and Cardiovascular Surgery, 124, 553-560. http://dx.doi.org/10.1067/mtc.2002.124670

[15] Sirivella, S., Gielchinsky, I. and Parsonnet, V. (2005) Results of Coronary Artery Endarterectomy and Coronary Artery Bypass Grafting for Diffuse Coronary Artery Disease. Annals of Thoracic Surgery, 80, 1738-1744. http://dx.doi.org/10.1016/j.athoracsur.2005.05.034

[16] Nurozler, F., et al. (2006) Off-Pump Coronary Endarterectomy in High-Risk Patients. Asian Cardiovascular and Thoracic Annals, 14, 227-230. http://dx.doi.org/10.1177/021849230601400312

[17] Vohra, H.A., et al. (2006) Early and Late Outcome after Off-Pump Coronary Artery Bypass Graft Surgery with Coronary Endarterectomy: A Single-Center 10-Year Experience. Annals of Thoracic Surgery, 81, 1691-1696. http://dx.doi.org/10.1016/j.athoracsur.2005.12.028

[18] Walley, V.M., Byard, R.W. and Keon, W.J. (1991) A Study of the Sequential Morphologic Changes after Manual Coronary Endarterectomy. Journal of Thoracic and Cardiovascular Surgery, 102, 890-894.

[19] Tiruvoipati, R., et al. (2005) Coronary Endarterectomy: Impact on Morbidity and Mortality When Combined with Coronary Artery Bypass Surgery. Annals of Thoracic Surgery, 79, 1999-2003. http://dx.doi.org/10.1016/j.athoracsur.2004.12.041

[20] Silberman, S., et al. (2002) Does Coronary Endarterectomy Increase the Risk of Coronary Bypass? Journal of Cardiac Surgery, 17, 267-271. http://dx.doi.org/10.1111/j.1540-8191.2001.tb01138.x

[21] Tiruvoipati, R., Loubani, M. and Peek, G. (2005) Coronary Endarterectomy in the Current Era. Current Opinion in Cardiology, 20, 517-520. http://dx.doi.org/10.1097/01.hco.0000182834.03402.43

[22] Schmitto, J.D., et al. (2009) Early Results of Coronary Artery Bypass Grafting with Coronary Endarterectomy for Severe Coronary Artery Disease. Journal of Cardiothoracic Surgery, 4, 52. http://dx.doi.org/10.1186/1749-8090-4-52

[23] Brenowitz, J.B., Kayser, K.L. and Johnson, W.D. (1988) Results of Coronary Artery Endarterectomy and Reconstruction. Journal of Thoracic and Cardiovascular Surgery, 95, 1-10.

[24] Minale, C., et al. (1989) Controversial Aspects of Coronary Endarterectomy. Annals of Thoracic Surgery, 48, $235-241$. http://dx.doi.org/10.1016/0003-4975(89)90077-5

[25] Edmunds Jr., L.H. (1993) Blood-Surface Interactions during Cardiopulmonary Bypass. Journal of Cardiac Surgery, 8, 
404-410. http://dx.doi.org/10.1111/j.1540-8191.1993.tb00384.x

[26] Schwann, T.A., et al. (2007) Survival and Graft Patency after Coronary Artery Bypass Grafting with Coronary Endarterectomy: Role of Arterial versus Vein Conduits. Annals of Thoracic Surgery, 84, 25-31.

http://dx.doi.org/10.1016/j.athoracsur.2007.02.053 